\title{
One Size Doesn't Fit All: Diversifying Data Science Course Projects by Student Background and Interests
}

\author{
Wensheng $\mathrm{Wu}$ \\ University of Southern California \\ wenshenw@usc.edu
}

\begin{abstract}
A key challenge to a data science program is how to tailor its curriculum to accommodate the needs and interests of students from diverse academic backgrounds. To address this challenge, we propose Proj4X, a general project model that gives students the flexibility in choosing subject areas for the projects based on their background and interests, while requiring every project to address the key knowledge and skills covered in the course. Although project-based learning has been well researched, only a few studies have addressed the challenges in managing student-initiated projects. We have applied Proj4X to a graduate database course in our data science program. We used student peer evaluation to measure the performance of projects objectively and study the effect of group size on the performance of projects and the correlation between specific aspects of a project and its overall rating. Student feedback shows that Proj4X can greatly foster the collaboration among students of diverse backgrounds and inspire them to work on a wide range of interesting topics: from satellite tracker, hospital search during pandemic, to wildlife diversity monitoring.
\end{abstract}

\section{CCS CONCEPTS}

- Information systems $\rightarrow$ Data management systems; • Social and professional topics $\rightarrow$ Computing education.

\section{KEYWORDS}

Data science; course project; diversification; student background

\section{ACM Reference Format:}

Wensheng Wu. 2021. One Size Doesn't Fit All: Diversifying Data Science Course Projects by Student Background and Interests . In 26th ACM Conference on Innovation and Technology in Computer Science Education V. 1 (ITiCSE 2021), fune 26-fuly 1, 2021, Virtual Event, Germany. ACM, New York, NY, USA, 7 pages. https://doi.org/10.1145/3430665.3456375

\section{INTRODUCTION}

Data science is an interdisciplinary field that has attracted students from very diverse academic backgrounds [7, 8, 25]. For example, among the 134 students taking the database course in our master's program on Applied Data Science in Fall 2020, 36\% majored in CS (including data science, software engineering, and computer engineering), 7\% in IT (e.g., IoT, digital media, and information

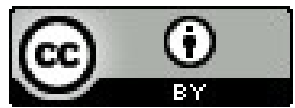

This work is licensed under a Creative Commons Attribution International 4.0 License. ITiCSE 2021, fune 26-fuly 1, 2021, Virtual Event, Germany.

(C) 2021 Copyright held by the owner/author(s).

ACM ISBN 978-1-4503-8214-4/21/06.

https://doi.org/10.1145/3430665.3456375 science), $24 \%$ in math or statistics, $20 \%$ in other engineering (e.g., mechanical and civil engineering), and $13 \%$ in other fields (e.g., economics and environmental science).

Many students have great passions in learning data science to tackle challenging problems in their fields or on the topics they are interested in. To better understand students' interests, we conducted a survey at the beginning of the semester, asking students to propose an area for their course projects and identify data science challenges in the area. Students enthusiastically responded to the survey.

For example, a student with an undergraduate major in Civil Engineering stated: "My current interests lie at the intersection of data science and public policy, especially transportation and environmental policy. I believe major challenges the transportation sector is facing today are related to traffic management and sustainability. Being able to use traffic data to identify hotspots and peak hours can address problems like congestion and pollution.”

While having students with such diverse backgrounds and interests poses serious challenges to course design, it also presents great opportunities for inclusive teaching and learning [30]. A key challenge is how to integrate the different perspectives of the students on data science into the curriculum development, in particular, the design of course projects-a critical part of learning experiences for data science students [7].

To address this challenge, we propose Proj4X, a project model that strikes a balance between foundation and diversity by giving the students the flexibility in choosing the subject area (X) for their projects (diversity), while requiring every project to address the key knowledge and skills covered in the course (foundation). It inspires the students to work on pressing problems the society is facing now, e.g., COVID-19 and climate change; and fosters collaborative learning by encouraging students from diverse backgrounds to work together on the projects.

Although there has been much research on project-based learning $[4,5,9,11,24,27]$, only a few studies have addressed the challenges in managing student-initiated projects. In particular, Pucher and Lehner [22] showed that students tended to be highly motivated when they were allowed to choose their own project topics. Ostrander et. al. [18] discussed the challenges in helping students find meaningful and relevant topics with appropriate scopes for their otherwise "open-ended" projects. Our work on Proj4X constitutes an important step towards systematically addressing these challenges (see more discussions in Related Work).

We have applied Proj4X to design projects for our database course. We conducted student peer evaluation to objectively assess the project quality, and designed a survey to further evaluate student learning outcomes and the effectiveness of the model. Here, we highlight some of the results. 
- Proj4X has enabled students to work on a wide range of interesting topics for their projects: from satellite tracker, traffic collision analysis, hospital search during pandemic, to wildlife diversity monitoring.

- Student peer evaluation indicates that 2-person teams performed much better than teams of 1 or 3 people on the average; and challenging data problems, interesting solutions, and intuitive UI were among the most important factors in determining the overall rating of the projects.

- $67 \%$ of students took pride in working on unique subject areas in their projects; and $71 \%$ of students agreed that Proj4X has helped keep the projects within the scope of the course (additional 25\% also agreed to a certain degree).

The rest of the paper is organized as follows. Section 2 discusses related work. Section 3 describes the Proj4X model and its application. Section 4 presents exemplary student projects developed using the model. Section 5 presents the student peer evaluation and learning outcomes. Finally, Section 6 concludes the paper.

\section{RELATED WORK}

We discuss related work from the following perspectives.

Computing for non-CS students: With the growing demands for computing courses from non-CS students [7], it is important to tailor the curriculum to appeal to students with diverse backgrounds $[8,25]$. Dawson et. al. [7] revamped CS1 for non-CS students to focus on how programming can be used in students' disciplines and how to design the projects so that students can apply program design skills to their own areas of interests. Bart et. al. [3] created a repository of over 40 datasets covering history, politics, medicine, and education, for the use of instructors in creating the curriculum for data science program. Dusen et. al. [29] created a new introductory course for undergraduate data science students to engage with students' interests and teach computational skills and concepts in connection with real-world issues and societal implications.

Project-based learning and sharing: Project-based learning has been widely used in computing courses to enhance students' hands-on experiences $[4,5,9,11,24,27]$. For example, Gorlatova et. al. [11] engaged a large number of students in a multi-year interdisciplinary research project and showed its effectiveness in helping students improve their collaboration skills.

Pérez and Rubio [19] rotated the roles of students playing in a software engineering project so that they experienced different challenges in different stages of the project. In Proj4X, roles of students in a project team are often determined by their backgrounds. For example, CS students in the team may lead the efforts on UI development, while non-CS students (e.g., biochemistry and math) may focus more on data modeling and analysis.

Miao et. al. [13] developed "ModelHub Discovery", an app for finding relevant data science projects on GitHub and DataHub for reuse. In Proj4X, we have used the projects on GitHub to inspire the students and asked students to post their projects on the web site for sharing. Tools like ModelHub Discovery may be used to facilitate the discovery and sharing of projects in Proj4X.

Student-initiated projects: Pucher and Lehner [22] reviewed more than 500 projects over 10 years and found out that if students suggested the projects, the motivation of the students was very

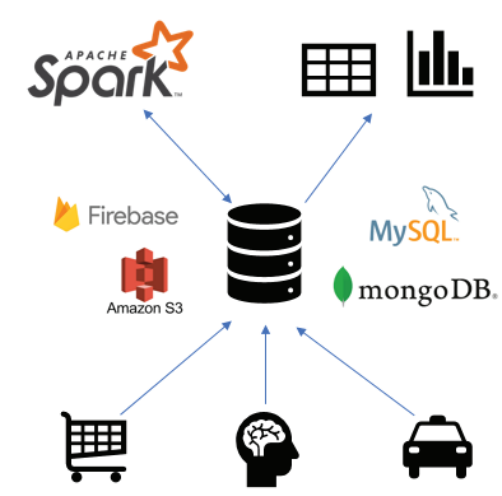

Figure 1: Project scheme based on the Proj4X model

high, and many projects where the origin of the idea came from students were very successful. The database systems course at MIT [17] provided students with a list of ideas for their course projects but also allowed students to choose their own project topics.

Ostrander et. al. [18] discussed the challenges faced by the instructors in supervising "open-ended" projects, such as "how to support students in the domain the instructors may not be familiar with?" and "how to help students find relevant projects with appropriate scopes?”. Proj4X addresses these challenges by providing students with a general project scheme (Figure 1), inspiring example projects and data problems, and clear requirements that keep the projects within the scope of the course.

\section{THE PROJ4X MODEL}

We now describe the Proj4X model in detail and show how to apply the model to design projects for the database course. The course covers data models, query languages, and query execution of SQL and NoSQL databases [23], and parallel data processing systems, such as Hadoop MapReduce [26] and Apache Spark [2].

The Proj4X model consists of the following four components.

(1) Foundation: The project needs to cover the key knowledge and skills that students are expected to learn and develop in the course. For example, we require every project in our database course to use a database for managing the data, utilize MapReduce systems (e.g., Apache Spark) for large-scale data processing or data analytics, and develop an intuitive interface for data retrieval and exploration.

(2) Diversification: Students can choose freely which subject areas and data sets to work on, which database systems (e.g., MySQL, Firebase [10], and MongoDB [14]) to use, what types of data problems (e.g., data modeling, cleaning, and transformation) to address, and what kinds of UI (e.g., webbased and mobile app) to develop in their projects.

(3) Inspiration: Students are advised to think about the pressing public health, environmental, and social problems we are facing now, and identify a real-world problem with societal impacts to address in their projects. Sample projects from past semesters and online resources (e.g., inspiring capstone projects) are used to motivate the students.

(4) Collaboration: Students are encouraged to work as a team and teams formed by students with diverse backgrounds are 

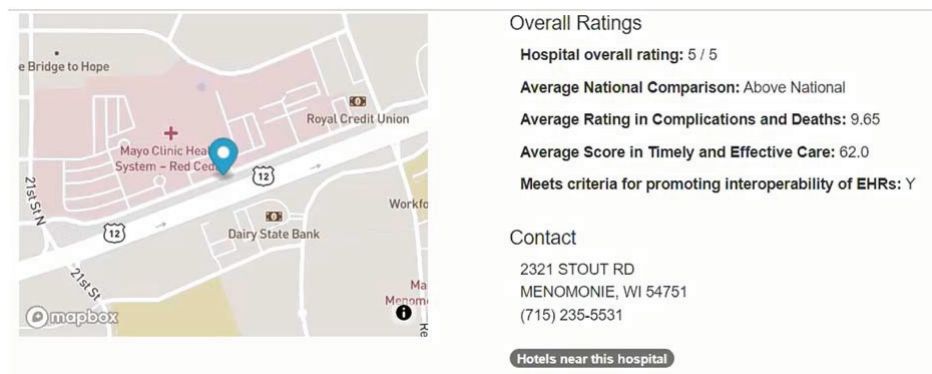

\begin{tabular}{llll} 
Name & Address & City & State \\
\hline $\begin{array}{l}\text { Best Western } \\
\text { Plus Eau Claire } \\
\begin{array}{l}\text { Conference } \\
\text { Center }\end{array}\end{array}$ & $\begin{array}{l}3340 \\
\text { Mondovi Rd }\end{array}$ & Eau Claire & WI \\
\hline Quality Inn Suites & $\begin{array}{l}3117 \text { Craig } \\
\text { Rd }\end{array}$ & Eau Claire & WI \\
\hline Royal Inn-hudson & $\begin{array}{l}1509 \text { Coulee } \\
\text { Rd }\end{array}$ & Hudson & WI \\
\hline
\end{tabular}

Project A1: Hospital location and ratings (left), and hotels near the hospital (right)
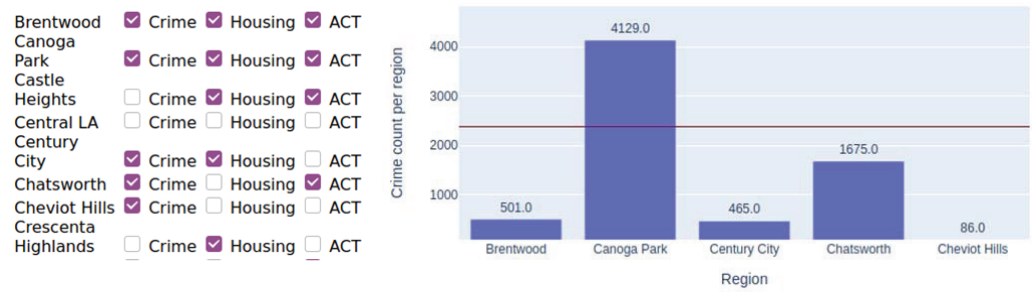

Project B1: Selecting neighborhoods in LA (left) and displaying their scores (right)

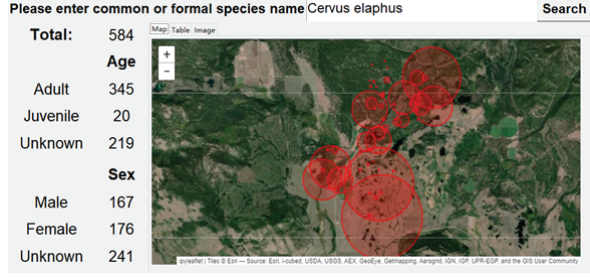

Project B6: Wildlife diversity monitoring

Figure 2: Exemplary student projects

considered favorably in assigning bonus points, e.g., when they have peer ratings similar to other teams. A multi-person team is also expected to meet higher expectations, e.g., using NoSQL and cloud databases, and integrating data from multiple data sources.

Figure 1 illustrates the project scheme for the course offered in Fall 2020. It highlights two key aspects of the Proj4X model: (1) foundation, e.g., requirements on using database (shown in the middle) and Spark (top-left); (2) diversification, e.g., flexible subject areas such as ecommerce, healthcare, and transportation (bottom), types of databases (e.g., Amazon S3 [1] and Firebase), and UI (topright). It also gives students a high-level picture on possible data flow between different components of projects. For example, data from diverse sources may first be integrated and then loaded into a database; next, Spark may be used to analyze the data in parallel; and finally, UI can fetch the data from the database for display.

Diverse data problems: Since many students were also taking machine learning and data visualization courses, we wanted the students to focus on the data management aspects (or "data problems") in their projects. For this, we motivated the students in class on possible data problems and provided an extensive list of example problems in the project guideline. For example,

- Data storage, e.g., using Amazon S3 or Firebase Storage to store (large) data objects in the cloud.

- Data modeling, e.g., structuring JSON data in Firebase.

- Data variety, e.g., handling images and time series data.

- Data cleaning, e.g., entity resolution and duplicate removal.

- Data transformation, e.g., converting JSON data into tables.

- Data integration, e.g., integrating traffic and weather data.

- Data aggregation, e.g., computing summary statistics of data.

- Data exploration, e.g., rollup and drill-down as in OLAP.

- Data retrieval, e.g., keyword search of NoSQL data.
- Parallel data processing, e.g., using Spark to perform largescale data cleaning, transformation, and integration.

Mini-capstone design: We encouraged students to take the course projects as a mini-capstone experience: based on their backgrounds and interests, design and implement an end-to-end project-from data collection, data management \& analysis, system development, to result presentation. We used a great variety of data science projects found online, e.g., SafeNav NYC [20] and pathways to graduation [21], to further motivate and inspire the students.

Fostering collaboration: We have implemented several strategies to foster the collaboration among the students, especially those from different backgrounds. (1) Ask students to introduce their backgrounds and interests in the first class meeting. (2) Conduct a background and course planning survey and help students in need find partners. (3) Use communication tools, such as Piazza and Slack, to connect the students offline. (4) Take the diversity of team into consideration when assigning reward points.

Interestingly, the first method turned out to be quite effective due to the online setting of the course. We have observed from student chats during the class that many students exchanged their contact information after the self-introduction. As we will see in Sections $4 \& 5$, many interesting and top-ranked projects were done by students from diverse backgrounds (e.g., biochemistry and CS).

\section{EXEMPLARY STUDENT PROJECTS}

In this section, we present examples of projects done by students in our database course. They are projects A1, B1, and B6 selected from the list of top-ranked projects shown in Figure 3 (ranking was based on student peer evaluation; see Section 4.1). Figure 2 shows the screenshots of the apps developed by these projects. At the end of each project, we also show the excerpts of learning experiences students stated in their final reports. 
Project A1: Keyword Driven Exploration of U.S. Hospitals and Hotels. This project was developed by two students. Student A has an undergraduate degree in chemistry and mathematics, and has worked at a biotechnology company for three years. Student B has a bachelor's degree in computer science, and was familiar with web app development and search engine technology.

The students were interested in working on healthcare domain given their backgrounds, and the project was motivated by observing the limited search functions on the Centers for Medicare \& Medicaid Services (CMS) web site [6]. To address the limitations, the students developed a hospital search app that has several interesting features: (1) It shows how the hospital compares to other hospitals in the nation, e.g., on patient experience. (2) It helps users find hotels near the hospital for temporary stay. (3) It displays a realtime tracking of local COVID-19 cases to help users make informed decisions during the pandemic.

The project integrated a hospital data set from CMS and a hotel review data set from Kaggle [12]. It used Apache Spark to precompute the average ratings for the hospitals and remove duplicate entries in the hotel data set in parallel. The project used MySQL to manage the hospital data, and Firebase to manage the hotel data.

Learning experiences: (1) Student A: This project was my first for many things-designing a database, making a web application, and collaborating with a partner virtually. (2) Student B: This project was my first for many things as well. I have learned how to choose different databases that work best for project needs. After a few meetings with the professor and my teammate, we chose to stick with MySQL and Firebase, and it turned out to be a smart choice. This choice allowed us to focus more on data problems such as data integration, data management, and entity resolution. It also allowed us to finish this project with a beautifully crafted website.

Project B1: Los Angeles Neighborhood Score. This project was done by a team of three students: one with background in industrial engineering and the other two in computer science. The team was inspired by the abundance of data publicly accessible on many city and state web sites, e.g., city of Los Angeles open data portal [16]. The goal of the project was to develop an app that allows users to compare the quality of life in different neighborhoods of LA by combining crime data from LA city web site, housing data from Zillow [31], and student ACT scores from the California Department of Education web site [15]. The app would be useful for people who want to explore or move into the city.

This project used MySQL on Amazon EC2 to store the original data, Spark SQL to compute aggregate scores for the neighborhoods, and Firebase to store the summary statistics for display on UI.

Learning experiences: This project proved to be a great source of learning for the team, as it focused on multiple datasets, from multiple sources, with multiple data processing and storage requirements allowed for the experimentation with a variety of tools. Selecting a realistic use case strongly contributed to the team's engagement and continued interest, as well as to the applicability of the learnings.

Project B6: Wildlife Diversity Monitoring. This project was developed by a student with an undergraduate degree in mathematics with concentration on data science. The student loves animals and wanted to develop an app that allows people to explore the images of wildlife dispersed over the Web. In the project, the student focused on the images of wildlife captured by the camera traps set up by the Montana Ranch Survey [28].

The project used Firebase to store the information about the cameras (e.g., locations), Firebase Storage to store the images captured by the cameras, and Spark to clean and integrate the data. The project has a number of interesting features, e.g., autocompletion to facilitate user searches, and a bubble map to intuitively show the number of images captured in different locations.

Learning experiences: For me, the most challenge part was the design of UI. Since my undergraduate major is math, I am more familiar with Python and MATLAB. Most programming assignments in my undergraduate were related to machine learning and optimization. So, I decided to use a Python package called tkinter to build UI, since tkinter has many examples and detailed documents.

\section{EVALUATION}

We now present the results of evaluating Proj4X in our database course in Fall 2020. The course ran for 14 weeks. It had three sections: section A has 43 students (20 project groups); section B 42 students (22 groups); section C 49 students (26 groups). Among all groups, 29 are 1-person, 14 2-person, and 25 3-person groups.

The projects were done in phases: group formation and proposals ( $10 \%$ of the project grade) due in the fourth week; midterm progress reports (10\%) in the eighth week; implementations (50\%), demos $(10 \%)$, and final reports $(20 \%)$ due in the last week. Students were asked to also describe their learning experiences and the responsibility of individual team members in the final reports. All members of the same group received the same grade. Bonus points were assigned based on student peer evaluation as discussed below.

In addition to the project, the course also had 5 homework assignments, weekly quizzes, 4 lab tasks that gave students hands-on experiences on big data software, a midterm, and a final exam.

\subsection{Student Peer Evaluation}

At the end of the semester, we asked students in each section to rate the quality of all other projects in the same section from 10 aspects. These aspects are listed in Figure 4. For example, "was the project topic interesting?", "did it address challenging data problems?", and "was its UI intuitive?". Each student also gave an overall rating to the project. The ratings on the individual aspects and the overall rating are all from 1 to 5 , with 5 being the best.

Based on student ratings and our detailed evaluation, we selected top-ranked projects (by their overall ratings) from each section to receive bonus points. Figure 3 shows the titles of these projects, the number of people in the groups, and the backgrounds of group members. We can see that many groups were formed by students from different backgrounds, e.g., chemistry, math, and CS. We can also see that student projects covered a great variety of subject areas, ranging from healthcare, aerospace, to public safety.

In addition, we analyzed the student evaluation data to find answers to the following questions.

(1) What was the effect of group size on the performance of groups? To see this, we normalized the (overall) rating, denoted as $X$, of a project into the standardized $Z$-score: $Z=(X-\mu) / \sigma$, where $\mu(\sigma)$ is the average (standard deviation) of project ratings in the 


\begin{tabular}{|c|l|c|l|}
\hline Section & Project Title & \# People & Background \\
\hline \multirow{4}{*}{ A } & 1. Keyword Driven Exploration of U.S. Hospitals and Hotels & 2 & Chemistry \& math, CS \\
\cline { 2 - 4 } & 2. Satellite Tracker & 1 & Geoinformatics \\
\cline { 2 - 4 } & 3. Ultimate Research Engine & 3 & Statistics, biostatistics, CS \\
\cline { 2 - 4 } & 4. Film Industry Plot (FiP) & 1 & Applied math, data science \& film \\
\cline { 2 - 4 } & 5. Clothing Style Search Engine & 2 & Math \& CS, economics \\
\hline \multirow{4}{*}{ B } & 1. Los Angeles Neighborhood Score & 3 & Industrial engineering, CS \\
\cline { 2 - 4 } & 2. Musiquity & 1 & CS \\
\cline { 2 - 4 } & 3. Reddit Streaming Data Analysis & 2 & Statistics \\
\cline { 2 - 4 } & 4. Los Angeles Traffic Collision and Weather & 3 & Software engineering, statistics, CS \\
\cline { 2 - 4 } & 5. Zillow-ish: Find Projected Home Values Near You! & 1 & Applied math \& data science \\
\cline { 2 - 4 } & 6. Wildlife Diversity Monitoring & 1 & Math \& data science \\
\hline \multirow{4}{*}{ C } & 1. Query translation & 2 & Spatial informatics, CS \\
\cline { 2 - 4 } & 2. Personalized Curriculum Recommendation & 3 & CS, CSE, management information system \\
\cline { 2 - 4 } & 3. COVID-19 Era Stock Trading Behaviors & 3 & Business \& statistics, biomedical engr, economics \\
\cline { 2 - 4 } & 4. DreamJob & 1 & Computer engineering \\
\cline { 2 - 4 } & 5. Integrating Genomics Data with Cancer Registry Records & 2 & Math, biochemistry \\
\hline
\end{tabular}

Figure 3: Projects that have received bonus points

\begin{tabular}{|c|c|c|c|c|c|c|c|c|c|c|}
\hline Section & $\begin{array}{c}\text { Interesting } \\
\text { topic }\end{array}$ & $\begin{array}{c}\text { Societal } \\
\text { impact }\end{array}$ & $\begin{array}{c}\text { Interesting } \\
\text { dataset }\end{array}$ & $\begin{array}{c}\text { Challenging } \\
\text { problems }\end{array}$ & $\begin{array}{c}\text { Interesting } \\
\text { solutions }\end{array}$ & $\begin{array}{c}\text { Spark } \\
\text { utilization }\end{array}$ & $\begin{array}{c}\text { Scale of } \\
\text { data }\end{array}$ & $\begin{array}{c}\text { Novel use } \\
\text { of DB }\end{array}$ & $\begin{array}{c}\text { Intuitive } \\
\text { UI }\end{array}$ & $\begin{array}{c}\text { UI wow } \\
\text { factors }\end{array}$ \\
\hline $\mathrm{A}$ & 0.63 & 0.6 & 0.68 & 0.7 & $\mathbf{0 . 7 2}$ & 0.65 & 0.66 & 0.7 & 0.66 & 0.7 \\
\hline $\mathrm{B}$ & 0.72 & 0.66 & 0.75 & $\mathbf{0 . 7 9}$ & 0.73 & 0.69 & 0.66 & 0.77 & 0.71 & 0.73 \\
\hline $\mathrm{C}$ & 0.51 & 0.66 & 0.72 & 0.72 & $\mathbf{0 . 7 4}$ & 0.68 & 0.68 & 0.73 & 0.73 & 0.58 \\
\hline
\end{tabular}

Figure 4: Correlation of specific aspects of a project with its overall rating

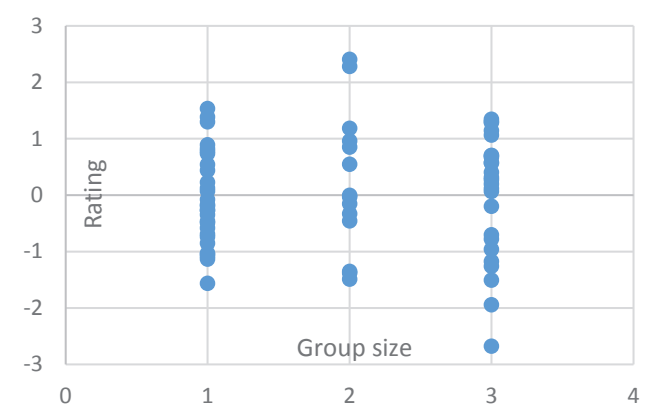

Figure 5: Rating vs group size

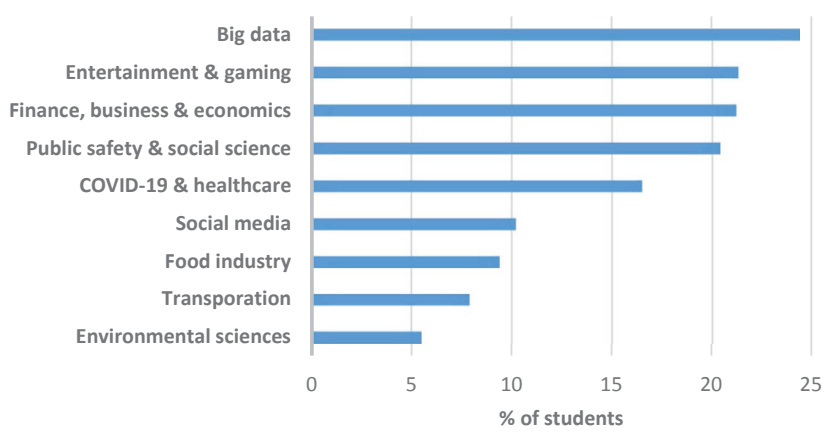

Figure 6: Major subject areas of student projects

same section. The normalization is to ensure that the ratings from different sections are comparable.

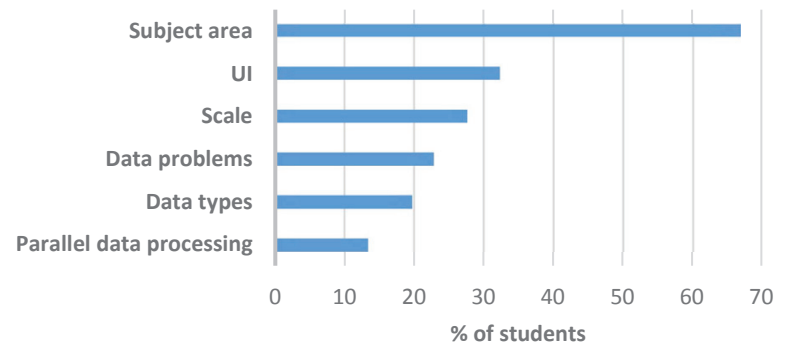

Figure 7: Unique aspects of projects suggested by students

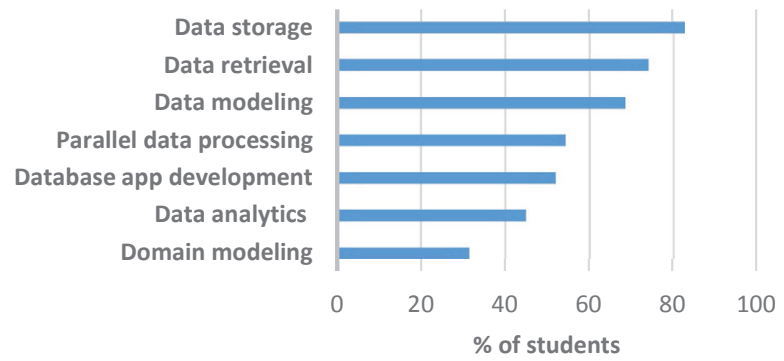

Figure 8: Key skills that projects helped acquire or enhance

Figure 5 plots the $Z$-scores of project groups against their sizes, where each dot represents a group. We can see that 2-person teams tend to perform better: the average $Z$-score of 2-person projects (.21) was much higher than that of 1-person (-.07) and 3-person 
projects (-.02). In fact, the highest-ranked projects in two sections (A1 and C1 in Figure 3) were done by two-person teams. Also a 3person project (on analyzing video game statistics, with all students from electrical engineering backgrounds) had the lowest rating over all sections, largely due to its poor UI design and subpar demo.

(2) Which aspects of the project affected the most on its overall rating? For this, we computed the Pearson correlation coefficient between the rating of individual aspect $(A)$ and the overall rating $(O)$ :

$$
\rho_{A, O}=\frac{\operatorname{cov}(A, O)}{\sigma_{A} \sigma_{O}}
$$

Figure 4 shows the results. We can see that the most important aspects (coefficient values shown in bold) are: (a) challenging data problems (which has the highest coefficient .79 in Section B) and (b) interesting solution to the problems (which has the highest coefficients in both Sections A and C). Novel use of databases and "wow" factors of UI also affected heavily the overall ratings.

\subsection{Learning Outcomes}

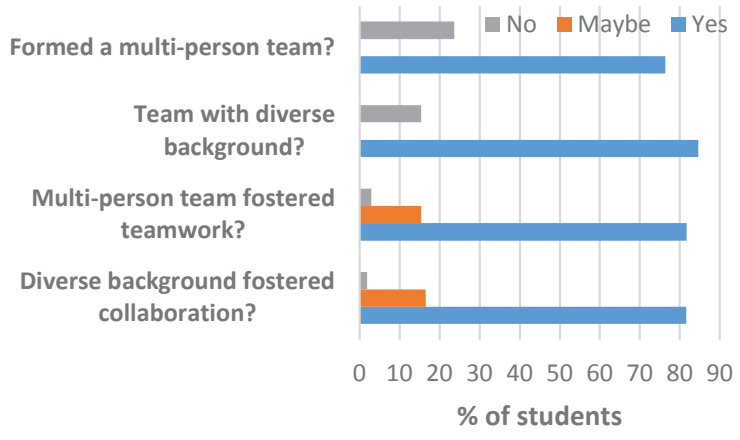

Figure 9: Collaboration fostered by the projects

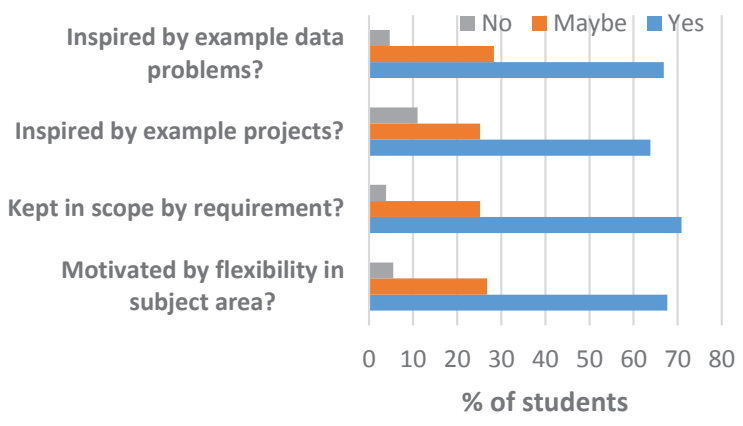

Figure 10: Project scope, motivation, and inspiration

In addition to the peer evaluation, we also conducted a survey (using Google Forms) to further understand students' learning outcomes. 127 students responded to the survey. The survey results are shown in Figure 6-10. Figure 6 shows the subject areas of projects ranked by the percentage of students working in the areas. Note that a project may cover multiple areas, e.g., addressing big data problem in social media. Figure 7 shows the unique aspects of their projects as suggested by the students. Figure 8 shows the key skills that the projects have helped students acquire or enhance. Figure 9 shows the collaboration fostered by the projects. Figure 10 shows the student feedback on project scope, motivation, and inspiration.

From the results, we can make the following observations:

- Students have worked on very diverse subject areas in their projects (Figure 6). This shows the effectiveness of Proj4X in diversifying student project topics (Section 3).

- $67 \%$ (85 out of 127) of students took pride in working on unique subject areas for their projects (Figure 7).

- The majority of the students felt that the projects have helped them acquire or enhance key data management skills including data storage, modeling, and retrieval (Figure 8). This shows the effectiveness of the foundation aspect of Proj4X.

- $85 \%$ of multi-person teams were formed by students from different backgrounds; and over $80 \%$ of students in multiperson teams agreed that the projects have greatly fostered the teamwork and collaboration (Figure 9).

- $71 \%$ of students agreed that the requirements in the project guideline have helped them keep the projects within the scope of the course. About 2/3 of students agreed that the provided example projects, data problems, and the flexibility in choosing subject areas have inspired and motivated them to work on the projects (Figure 10). Note that there were also about $20-30 \%$ of students who agreed to a certain degree.

\subsection{Discussions}

A/B testing: To further evaluate Proj4X, we may also divide students into two groups: one group works on the topics assigned by the instructor and the other on the topics chosen by students using the Proj4X model. It would be interesting to compare the motivations and learning experiences of students in the two groups.

Individual contributions: We evaluated the performance of individual members of a project team based on the division of tasks stated in the proposal and the completion of these tasks shown in demo and final report. However, we regarded a multi-person project as a teamwork and did not grade individual members separately.

Workload: All multi-person teams were required to use at least two databases in their projects: one relational and the other NoSQL. In addition, 3-person teams were required to use a cloud-based NoSQL database, properly integrate data in the two databases, and present the data in a unified interface.

\section{CONCLUSIONS \& FUTURE DIRECTION}

We have presented Proj4X, a unique project design model that balances the foundation of course and the diversity of students, inspires the students, and fosters the collaboration among the students. The evaluation results indicate that Proj4X has enabled students to develop many interesting projects covering a wide range of subject areas, while making sure that students obtain hands-on experiences on the key knowledge and skills covered in the course. Although we discuss Proj4X in the context of data science courses, we believe its idea is generally applicable to other courses with diverse students.

Besides further evaluation of Proj4X on diverse courses of our data science program, we are also investigating the direction of inviting the faculty in the disciplines related to student projects (e.g., public health) to co-advise the students on their projects (e.g., via guest lectures and project group meetings). 


\section{REFERENCES}

[1] Amazon. 2020. Amazon Simple Storage Service (S3). https://aws.amazon.com/s3/.

[2] Michael Armbrust, Reynold S Xin, Cheng Lian, Yin Huai, Davies Liu, Joseph K Bradley, Xiangrui Meng, Tomer Kaftan, Michael J Franklin, Ali Ghodsi, et al. 2015. Spark sql: Relational data processing in spark. In Proceedings of the 2015 ACM SIGMOD international conference on management of data. 1383-1394.

[3] Austin Cory Bart, Ryan Whitcomb, Dennis Kafura, Clifford A Shaffer, and Eli Tilevich. 2017. Computing with corgis: Diverse, real-world datasets for introductory computing. ACM Inroads 8, 2 (2017), 66-72.

[4] Assma Boughoula, Aaron Green, and ChengXiang Zhai. 2020. Collective Development of Large Scale Data Science Products via Modularized Assignments: An Experience Report. In Proceedings of the 51st ACM Technical Symposium on Computer Science Education. 1200-1206.

[5] Stefan C Christov and Mark E Hoffman. 2019. Experiential Learning of Software Project Management and Software Development via Course Collaboration. In Proceedings of the 50th ACM Technical Symposium on Computer Science Education. $160-166$.

[6] CMS. 2020. Centers for Medicare \& Medicaid Services. https://www.cms.gov/.

[7] Jessica Q Dawson, Meghan Allen, Alice Campbell, and Anasazi Valair. 2018 Designing an introductory programming course to improve non-majors' experiences. In Proceedings of the 49th ACM Technical Symposium on Computer Science Education. 26-31.

[8] Debzani Deb, Russell M Smith, and Muztaba Fuad. 2019. Infusing Data Science Across Disciplines. In Proceedings of the 2019 ACM Conference on Innovation and Technology in Computer Science Education. 302-302.

[9] Maria Lydia Fioravanti, Bruno Sena, Leo Natan Paschoal, Laíza R Silva, Ana P Allian, Elisa Y Nakagawa, Simone RS Souza, Seiji Isotani, and Ellen F Barbosa 2018. Integrating project based learning and project management for software engineering teaching: An experience report. In Proceedings of the 49th ACM Technical Symposium on Computer Science Education. 806-811.

[10] Google. 2020. The Firebase realtime database. https://firebase.google.com/.

[11] Maria Gorlatova, John Sarik, Peter Kinget, Ioannis Kymissis, and Gil Zussman. 2013. Project-based learning within a large-scale interdisciplinary research effort. In Proceedings of the 18th ACM conference on Innovation and technology in computer science education. 207-212.

[12] Kaggle. 2020. Kaggle: Your Home for Data Science. https://www.kaggle.com/.

[13] Hui Miao, Ang Li, Larry S Davis, and Amol Deshpande. 2017. On model discovery for hosted data science projects. In Proceedings of the 1st Workshop on Data Management for End-to-End Machine Learning. 1-4.

[14] MongoDB. 2020. The MongoDB database. https://mongodb.com/.
[15] California Dept of Education. 2020. Postsecondary Preparation. https://www.cde.ca.gov/ds/sp/ai/.

[16] City of Los Angeles. 2020. Los Angeles Open Data Portal. https://data.lacity.org/.

[17] MIT OpenCourseWare. 2010. Database Systems (course project). https://ocw.mit.edu/courses/electrical-engineering-and-computer-science/6830-database-systems-fall-2010/assignments/project.

[18] Tina Ostrander, Ruby ElKharboutly, and Karen Jin. 2017. Surviving" Open-ended Projects" in Project-Based Learning: A Teacher's Perspective. In SIGCSE. 727-727.

[19] Beatriz Pérez and Ángel L Rubio. 2020. A project-based learning approach for enhancing learning skills and motivation in software engineering. In Proceedings of the 51st ACM Technical Symposium on Computer Science Education. 309-315.

[20] UC Berkeley Data Science Capstone Project. 2020. SafeNav NYC. https://www.ischool.berkeley.edu/projects/2020/safenav.

[21] UW-Madison Data Science Capstone Project. 2020. Pathways to Graduation. https://datasciencedegree.wisconsin.edu/capstone-projects/pathways-tograduation-msds-edition/.

[22] Robert Pucher et al. 2011. Project based learning in computer science-a review of more than 500 projects. Procedia-Social and Behavioral Sciences 29 (2011).

[23] Pramod J Sadalage and Martin Fowler. 2013. NoSQL distilled: a brief guide to the emerging world of polyglot persistence. Pearson Education.

[24] Sreecharan Sankaranarayanan. 2019. Online Mob Programming: Effective Collaborative Project-Based Learning. In Proceedings of the 50th ACM Technical Symposium on Computer Science Education. 1296-1296.

[25] Sadia Sharmin. 2020. Open-Ended Exercises in CS1: The Impact on Female, NonMajor and Inexperienced Computer Science Students. In Proceedings of the 2020 ACM Conference on Innovation and Technology in Computer Science Education. 558-558.

[26] Konstantin Shvachko, Hairong Kuang, Sanjay Radia, and Robert Chansler. 2010. The hadoop distributed file system. In 2010 IEEE 26th symposium on mass storage systems and technologies (MSST). Ieee, 1-10.

[27] Kalpathi Subramanian and Kiran Budhrani. 2020. Influence of Course Design on Student Engagement and Motivation in an Online Course. In Proceedings of the 51st ACM Technical Symposium on Computer Science Education. 303-308.

[28] Montana Ranch Survey. 2020. https://emammal.si.edu/montana-ranch-survey.

[29] Eric Van Dusen, Anthony Suen, and Cathryn Carson. 2020. Innovation in Undergraduate Data Science Education. In Proceedings of the 51st ACM Technical Symposium on Computer Science Education. 1397-1397.

[30] Wensheng Wu. 2021. SQL2X: Learning SQL, NoSQL, and MapReduce via Translation. In Proceedings of the 52nd ACM Technical Symposium on Computer Science Education. 590-596.

[31] Zillow. 2020. https://www.zillow.com/research/data/. 